\title{
A Linear Algebraic Approach for Loss Tomography in Mesh Topologies Using Network Coding
}

\author{
Jiaqi Gui, Vahid Shah-Mansouri, and Vincent W.S. Wong \\ Department of Electrical and Computer Engineering \\ The University of British Columbia, Vancouver, Canada \\ E-mail: $\{$ jiaqig, vahids, vincentw\} @ece.ubc.ca
}

\begin{abstract}
Loss tomography aims to infer link loss rates using end-to-end measurements. We investigate active loss tomography on mesh topologies. When network coding is applied, based on the content of the received probe packet, a receiver should distinguish which paths have successfully transmitted a probe and which paths have not. We establish a lower bound on probe size which is necessary for obtaining such end-to-end observations. Furthermore, we propose a linear algebraic (LA) approach to developing consistent estimators of link loss rates. Our approach exploits the inherent correlation between the losses on links and the losses on different sets of paths, so that the estimators converge to the actual loss rates as the number of probes increases. We also prove that the identifiability of a link is a necessary and sufficient condition for the consistent estimation of its loss rate. Simulation results show that the LA approach achieves better estimation accuracy than the belief propagation (BP) algorithm, after sending reasonably sufficient probes.
\end{abstract}

\section{INTRODUCTION}

Accurate and efficient measurement of network-internal characteristics is critical for monitoring and managing of largescale networks. However, the lack of centralized control of modern networks makes it prohibitively expensive to access a wide range of routers to gain packet-level statistics. Network tomography provides an alternative approach. It collects and analyzes end-to-end measurements to infer link loss, delay and network topology [1]-[3]. Instead of passively monitoring data flow, active tomography sends probe packets from sources to receivers located on the periphery of the network to obtain informative and reliable path-level measurements.

In this paper, we consider the problem of link loss tomography on mesh topologies in wired networks. Although there are extensive studies of link loss inference on multicast tree topologies [4]-[6], loss tomography on mesh topologies is a challenging problem. One existing approach is to use multiple trees to cover a mesh topology and combine the inferred loss rates [7]. However, this approach would result in two drawbacks. The first one is its low bandwidth efficiency. Those links as part of multiple trees would be traversed by multiple probe packets in each time slot, which creates additional traffic. The second drawback is the high monitoring cost for the deployment of receivers in each multicast tree.

Applying network coding in loss tomography can increase bandwidth efficiency [8]-[10]. In a network which is capable of performing network coding in addition to multicast, the intermediate nodes linearly combine incoming probe packets and forward the coded probe packets to the outgoing links.
An instructive example is illustrated in [10] that each link in a mesh topology can be traversed in each time slot by exactly one probe. Besides the number of probes on each link, the probe size also directly affects bandwidth efficiency. We establish a lower bound on probe size. Based on the content of one received probe packet, a receiver should distinguish which paths have successfully transmitted a probe and which paths have not. The lower bound is necessary for obtaining such valid end-to-end observations.

To reduce monitoring cost, a set of end-to-end paths only requires a limited number of sources and receivers. Nonetheless, the existing inference approaches have not fully utilized the end-to-end observations. A belief propagation (BP) algorithm for link loss rate inference in wireless sensor networks is proposed in [11]. The BP algorithm is combined with the use of network coding in [10]. The approach in [12] first estimates the number of faulty links on a path, then uses the global information to determine loss rates to the links. It requires a priori probability distribution function on link loss rates (the global information) and the majority of links being lossless. On the contrary, we propose a linear algebraic (LA) approach that exploits the inherent correlation between the losses on links and those on different sets of paths. As a result, the proposed estimators converge to the actual loss rates as the number of probes increases.

The contributions of our work are summarized as follows:

- We establish a lower bound on probe size, which is necessary for obtaining valid end-to-end observations when network coding is applied.

- We propose an LA approach to developing consistent estimators of link loss rates, which converge in probability to the actual loss rates as the number of probes increases.

- We prove that the identifiability of a link, which only depends on the network topology, is a necessary and sufficient condition for the consistent estimation of its loss rate.

- Simulation results show that the LA approach achieves better estimation accuracy than the BP algorithm, after sending reasonably sufficient probes. Besides, we can use a small number of sources and flexibly choose their locations in practice, using the proposed approach.

The remainder of this paper is organized as follows. In Section II, we present the system model. In Section III, we 
establish a lower bound on probe size when network coding is applied. Section IV presents an LA approach to developing consistent estimators of link loss rates. Simulation results are discussed in Section V. We conclude this paper in Section VI.

\section{SYSTEM MODEL}

We model the network as a directed acyclic graph $\mathcal{G}=$ $(\mathcal{V}, \mathcal{E})$, consisting of a set of nodes $\mathcal{V}$ and a set of links $\mathcal{E}$. The node set $\mathcal{V}$ includes routers and periphery devices where probe packets are sent and received. A link $e \in \mathcal{E}$ denotes a directed communication link that connects two network nodes.

Let $\mathcal{S}$ and $\mathcal{R}$ denote the set of source nodes and the set of receiver nodes, respectively. The set of monitored end-to-end paths is denoted by $\mathcal{P}$. A path $P \in \mathcal{P}$ is a set of directed links from a source to a receiver. Let $\mathcal{P}(e)$ denote the set of paths that include link $e$. For the clarity of discussion, we also represent a link or a path by the sequence of nodes it traverses.

We define a path-link matrix $\mathbf{M}=\left(m_{i, j}\right)_{|\mathcal{P}| \times|\mathcal{E}|}$, whose $|\mathcal{P}|$ rows correspond to the $|\mathcal{P}|$ paths and the $|\mathcal{E}|$ columns correspond to the $|\mathcal{E}|$ links, as follows: The element $m_{i, j}$ is equal to 1 if the $i$ th path in $\mathcal{P}$ includes the $j$ th link in $\mathcal{E}$, and is equal to 0 otherwise.

As an example, the directed acyclic graph in Fig. 1 has three paths from source $s$ to receiver $r$. Its path-link matrix is a 3 by 7 binary matrix as shown below:

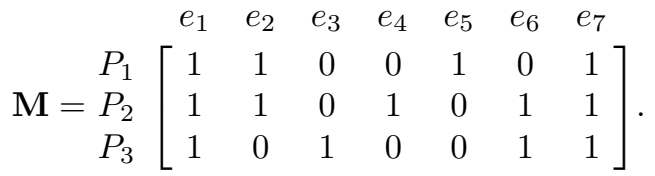

Given a directed acyclic graph $\mathcal{G}=(\mathcal{V}, \mathcal{E})$ and a set of monitored end-to-end paths $\mathcal{P}$, a link $e \in \mathcal{E}$ is called identifiable, if for each link pair $\left(e, e^{\prime}\right)$ where $e^{\prime} \in \mathcal{E} \backslash\{e\}$, there exists at least one path in $\mathcal{P}$ that includes exactly one of the two links, i.e, $\mathcal{P}(e) \neq \mathcal{P}\left(e^{\prime}\right)$. As in Fig. $1, e_{2}, e_{3}, \ldots, e_{6}$ are identifiable links, while $e_{1}$ and $e_{7}$ are non-identifiable links. The following proposition shows that the identifiability of a link, which only depends on the network topology, is a necessary condition for the estimation of its loss rate.

Proposition 1: The loss rate of a link can be estimated only if the link is an identifiable link.

Proof: We prove it by contradiction. Suppose there exists a link pair $\left(e, e^{\prime}\right)$ where $e, e^{\prime} \in \mathcal{E}$ such that all paths in $\mathcal{P}$ includes either both or none of them, i.e., $\mathcal{P}(e)=\mathcal{P}\left(e^{\prime}\right)$. If a probe packet is dropped on either link $e$ or $e^{\prime}$, the same end-toend observation would be obtained in either case. Therefore, we cannot diagnose on which link the loss of probe packet occurs and it is not possible estimate the loss rate of these links.

For an identifiable link $e, \mathcal{P}(e)$ is different from other $\mathcal{P}\left(e^{\prime}\right)$. We refer to the non-identifiable links that are included in the same paths as one virtual link. As in Fig. $1, e_{1}$ and $e_{7}$ are referred as one virtual link $e_{v_{1}}$. Let $\mathcal{E}_{I}$ and $\mathcal{E}_{V}$ denote the set of identifiable links and the set of virtual links, respectively. Thus, for each link $e \in \mathcal{E}_{I} \cup \mathcal{E}_{V}, \mathcal{P}(e)$ is different from other $\mathcal{P}\left(e^{\prime}\right)$.

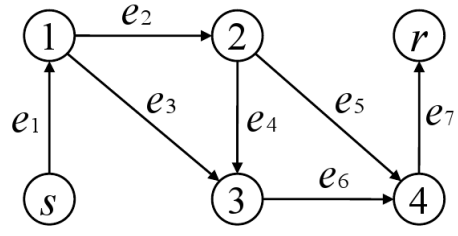

Fig. 1. A directed acyclic graph with three paths from source $s$ to receiver

We fix the order of elements in $\mathcal{E}_{I} \cup \mathcal{E}_{V}$. Accordingly, we define a modified path-link matrix (type 1) $\overline{\mathbf{M}}=$ $\left(\bar{m}_{i, j}\right)_{|\mathcal{P}| \times\left|\mathcal{E}_{I} \cup \mathcal{E}_{V}\right|}$ as follows: The element $\bar{m}_{i, j}$ is equal to 1 if the $i$ th path in $\mathcal{P}$ includes the $j$ th link in $\mathcal{E}_{I} \cup \mathcal{E}_{V}$, and is equal to 0 otherwise.

The type 1 modified path-link matrix for the graph in Fig. 1 is shown below:

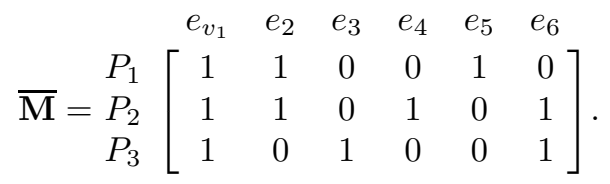

We model the loss of packets on different links by a set of mutually independent Bernoulli processes. Losses are therefore spatial and temporal independent. This model has also been used in the literature [4]-[7], [11], [12].

We define $\alpha_{j} \in(0,1]$ as the link success rate of the $j$ th link in set $\mathcal{E}_{I} \cup \mathcal{E}_{V}$, which is the probability that a packet can be successfully transmitted on the link. Thus, $1-\alpha_{j}$ denotes the loss rate of the link. Moreover, we define $\beta_{i} \in(0,1]$ as the path success rate of the $i$ th end-to-end path in set $\mathcal{P}$, which is the probability that a probe packet can be successfully transmitted on the path.

Unlike data packets, a probe would not be retransmitted if it is dropped in our framework. Thus, we have

$$
\prod_{j=1}^{\left|\mathcal{E}_{I} \cup \mathcal{E}_{V}\right|}\left(\alpha_{j}\right)^{\bar{m}_{i, j}}=\beta_{i}, \quad i=1, \ldots,|\mathcal{P}| .
$$

Taking logarithm on both sides of (3), we can reformulate it to linear equations as follows:

$$
\sum_{j=1}^{\left|\mathcal{E}_{I} \cup \mathcal{E}_{V}\right|} \bar{m}_{i, j} \log \left(\alpha_{j}\right)=\log \beta_{i}, \quad i=1, \ldots,|\mathcal{P}| .
$$

Setting $a_{j}=\log \alpha_{j}$ and $b_{i}=\log \beta_{i}$, (4) becomes

$$
\sum_{j=1}^{\left|\mathcal{E}_{I} \cup \mathcal{E}_{V}\right|} \bar{m}_{i, j} a_{j}=b_{i}, \quad i=1, \ldots,|\mathcal{P}| .
$$

We define two column vectors $\mathbf{a}=\left(a_{j}\right)_{\left|\mathcal{E}_{I} \cup \mathcal{E}_{V}\right| \times 1}$ and $\mathbf{b}=$ $\left(b_{i}\right)_{|\mathcal{P}| \times 1}$. The system can be represented in the matrix form

$$
\overline{\mathbf{M}} \mathbf{a}=\mathbf{b} .
$$

Path success rates can usually be inferred by end-to-end observations, which yields $\hat{\mathbf{b}}$, the estimator of $\mathbf{b}$. The column 
vector â remains unknown. Thus, (6) becomes a system of $|\mathcal{P}|$ equations with $\left|\mathcal{E}_{I} \cup \mathcal{E}_{V}\right|$ unknowns as follows:

$$
\overline{\mathbf{M}} \hat{\mathbf{a}}=\hat{\mathbf{b}} .
$$

In most cases, there are more identifiable and virtual links than paths, so that (7) is under-determined.

We build the inference framework in two phases. In the first phase, we apply network coding and perform end-to-end measurements. $n$ batches of probe packets are sent from the sources in a synchronized manner. In each time slot, the intermediate nodes linearly combine incoming probes according to the coding coefficients. In the second phase, we inspect the contents of the received probe packets that provide more information than path success rates. We apply linear algebraic approach to consistently estimate link loss rates using such information. In next two sections, we address the issues in the two phases, respectively.

\section{Probe Coding Schemes}

We refer to probe packets and network coding schemes jointly as probe coding schemes. A probe coding scheme is valid if we can determine which paths have successfully transmitted a probe and which paths have not, from the endto-end observations. We adopt linear network coding schemes that are sufficient for our task. In this section, we establish a lower bound on the probe size (i.e., number of bits in each probe packet), which is necessary for a valid probe coding scheme.

A probe packet is a binary vector $(\cdot)_{2}$ of length $\ell$, which can be interpreted as an element in a finite field $\mathbb{F}_{q}$ with an alphabet of size $q\left(q=2^{\ell}\right)$. A coding coefficient can also be interpreted as an element in the finite field $\mathbb{F}_{q}$. Within valid probe coding schemes, the probe size $\ell$ is desired to be as small as possible, since it is directly related to bandwidth efficiency.

Let $(h, r) \in \mathcal{E}$ denote a link adjacent to a receiver $r \in \mathcal{R}$. Let $\mathcal{P}(h, r)$ denote the set of end-to-end paths that include link $(h, r)$. If $r$ has multiple incoming links, it would know from which link the received probes are. The following theorem shows a lower bound on probe size.

Theorem 1: Let a directed acyclic graph $\mathcal{G}=(\mathcal{V}, \mathcal{E})$ be given with a set of monitored end-to-end paths $\mathcal{P}$. For valid probe coding schemes, the size of the probes transmitted on the paths in $\mathcal{P}(h, r)$ satisfies $\ell \geq|\mathcal{P}(h, r)|$.

Proof: $\mathcal{P}(h, r)=\left\{P_{1}, P_{2}, \ldots, P_{|\mathcal{P}(h, r)|}\right\}$. For valid probe coding schemes, based on a received probe, a receiver $r$ should distinguish which paths have successfully transmitted a probe and which paths have not. Without loss of generality, we start from $P_{1}$. Since a zero binary vector will introduce ambiguity, $(1)_{2}$ is the smallest binary vector we can use to denote the case where only $P_{1}$ has successfully transmitted a probe. $(10)_{2}$ is the smallest binary vector we can use to denote the case where only $P_{2}$ has successfully transmitted a probe. Since $(11)_{2}$ denotes the case where both $P_{1}$ and $P_{2}$ have successfully transmitted a probe, $(100)_{2}$ is the smallest binary vector we can use to denote the case where only $P_{3}$ has successfully transmitted a probe. By induction, we can show that $(10 \ldots 0)_{2}$ of length $|\mathcal{P}(h, r)|$ is the smallest binary vector we can use to denote the case where only $P_{|\mathcal{P}(h, r)|}$ has successfully transmitted a probe. Then, we modify the above binary vectors to vectors of length $|\mathcal{P}(h, r)|$ with zeros added to the left-hand side. Thus, for the probes transmitted on the paths in $\mathcal{P}(h, r)$, we have $\ell \geq|\mathcal{P}(h, r)|$ and $q \geq 2^{|\mathcal{P}(h, r)|}$.

It remains uncertain that under what conditions the lower bound on probe size is achievable. The formulated procedure of finding a valid coding scheme will help solving the problem mentioned above. So far, we only illustrate how we find a valid probe coding scheme in some simple topologies.

Example 1: We consider a directed acyclic graph $\mathcal{G}=$ $(\mathcal{V}, \mathcal{E})$ depicted in Fig. 1.

We select intermediate nodes with multiple incoming links as the nodes performing network coding, which would be nodes 3 and 4 . We initially choose their coding coefficients as $[1,1]$. The remaining intermediate nodes multicast probes. We verify that $|\mathcal{P}(4, r)|=3$. By Theorem 1 , the lower bound on probe size is 3 bits. We choose probes over a finite field $\mathbb{F}_{2^{3}}$. Thus, the source sends $(001)_{2}$ to outgoing link $(s, 1)$.

Suppose only $P(s, 1,2,4, r)$ has successfully transmitted a probe. Then, $r$ would receive $(001)_{2}$. Suppose only $P(s, 1,2,3,4, r)$ has successfully transmitted a probe. Then, $r$ would also receive $(001)_{2}$. Let node 4 use coding coefficients $[2,1]$, so that $r$ would receive $(010)_{2}$ for $P(s, 1,2,3,4, r)$. Suppose only $P(s, 1,3,4, r)$ has successfully transmitted a probe. Then, $r$ would also receive $(010)_{2}$. Let node 3 use coding coefficients $[2,1]$, so that $r$ would receive $(100)_{2}$ for $P(s, 1,3,4, r)$. A received probe packet with multiple 1 's represents that a batch of probes has been successfully transmitted on multiple paths.

As we can see, this is a valid probe coding scheme and the lower bound on probe size is achievable in this example.

\section{Linear Algebraic Approach}

As described previously, the system in (7) has $|\mathcal{P}|$ equations with $\left|\mathcal{E}_{I} \cup \mathcal{E}_{V}\right|$ unknowns. However, $|\mathcal{P}|$ may be less than $\left|\mathcal{E}_{I} \cup \mathcal{E}_{V}\right|$, such as the topology in Fig. 1. Even when $|\mathcal{P}| \geq$ $\left|\mathcal{E}_{I} \cup \mathcal{E}_{V}\right|$, it does not ensure that â in (7) can be determined. In this section, we propose a linear algebraic (LA) approach and show that â can be determined by least-squares [13].

By inspecting the content of received probe packets, we can estimate not only the success rate of a single path, but also the success rate of any combination of paths. This is unique to networks with probe coding schemes and cannot be achieved by only routing probes. We denote the power set of $\mathcal{P}$ by $\mathscr{P}$. Thus, $|\mathscr{P}|=2^{|\mathcal{P}|}$. Each element of $\mathscr{P}$ is a subset of $\mathcal{P}$, which can be used to represent different combinations of paths.

Let $\theta_{i} \in\left\{\theta_{1}, \theta_{2}, \ldots, \theta_{|\mathscr{P}|-1}\right\}$ denote the path set success rate of the $i$ th path set in $\mathscr{P} \backslash\{\varnothing\}$, which is the probability that a probe (or a batch of probes) can be successfully transmitted on the path (or all the paths) in the $i$ th path set. We define a path set success rate except for $\varnothing \in \mathscr{P}$, because we require the probability that at least one path can successfully transmit a probe to obtain an equation with link success rates and a path set success rate. 
Accordingly, we define a modified path-link matrix (type 2) $\widetilde{\mathbf{M}}=\left(\widetilde{m}_{i, j}\right)_{(|\mathscr{P}|-1) \times\left|\mathcal{E}_{I} \cup \mathcal{E}_{V}\right|}$ as follows: The element $\widetilde{m}_{i, j}$ is equal to 1 if there exists a path in the $i$ th path set in $\mathscr{P} \backslash\{\varnothing\}$ which includes the $j$ th link in $\mathcal{E}_{I} \cup \mathcal{E}_{V}$, and is equal to 0 otherwise.

The type 2 modified path-link matrix for the graph in Fig. 1 is shown below:

$$
\widetilde{\mathbf{M}}=\left[\begin{array}{cccccc}
1 & 1 & 0 & 0 & 1 & 0 \\
1 & 1 & 0 & 1 & 0 & 1 \\
1 & 0 & 1 & 0 & 0 & 1 \\
\hdashline 1 & 1 & 0 & -1 & 1 & -1 \\
1 & 1 & 1 & 0 & 1 & 1 \\
1 & 1 & 1 & 1 & 0 & 1 \\
1 & 1 & 1 & 1 & 1 & 1
\end{array}\right]
$$

We define a column vector, $\mathbf{c}=\left(c_{i}\right)_{(|\mathscr{P}|-1) \times 1}$, where $c_{i}=$ $\log \theta_{i}$. The column vector a is defined as in Section II. Thus, we have a linear system

$$
\sum_{j=1}^{\left|\mathcal{E}_{I} \cup \mathcal{E}_{V}\right|} \widetilde{m}_{i, j} a_{j}=c_{i}, \quad i=1, \ldots,|\mathscr{P}|-1
$$

or in the matrix form

$$
\widetilde{\mathrm{M}} \mathbf{a}=\mathbf{c} .
$$

For each path set in $\mathscr{P} \backslash\{\varnothing\}$, the $n$ probe batches sent can be considered as a binomial experiment consisting of $n$ trials. The associated binomial random variable $X_{i}$ is defined as the number of received probes whose contents represent that a probe (or a batch of probes) has been successfully transmitted on the path (or all the paths) in the $i$ th path set.

The sample proportion $\hat{\theta}_{i}=X_{i} / n$ is a maximum likelihood estimator (MLE) of $\theta_{i}$ [14] (or an ML estimate resulting from end-to-end measurement $x_{i}$ substituted in the place of $X_{i}$ ). Accordingly, we can have $\hat{\mathbf{c}}$, the estimator of $\mathbf{c}$. The column vector â remains unknown. Thus, we extend (7) to a system of $|\mathscr{P}|-1$ equations with $\left|\mathcal{E}_{I} \cup \mathcal{E}_{V}\right|$ unknowns, as follows:

$$
\widetilde{\mathbf{M}} \hat{\mathbf{a}}=\hat{\mathbf{c}} .
$$

The above system has more equations than unknowns, i.e.,

$$
|\mathscr{P}|-1 \geq\left|\mathcal{E}_{I} \cup \mathcal{E}_{V}\right|
$$

This is because for each link $e \in \mathcal{E}_{I} \cup \mathcal{E}_{V}, \mathcal{P}(e)$ is different from other $\mathcal{P}\left(e^{\prime}\right)$, while $|\mathcal{P}|$ paths can have at most $2^{|\mathcal{P}|}-1$ different combinations of paths. (12) is a necessary condition for â to be determined.

To prove that $\hat{\mathbf{a}}$ in (11) can be determined by least-squares, we introduce a $(|\mathscr{P}|-1) \times(|\mathscr{P}|-1)$ auxiliary matrix $\mathcal{M}(|\mathcal{P}|)$ of a type 2 modified path-link matrix $\widetilde{\mathbf{M}}$ with an end-to-end path set $\mathcal{P}$. Some new columns are added to $\mathcal{M}(|\mathcal{P}|)$ such that the $|\mathscr{P}|-1$ column vectors in the top $|\mathcal{P}| \times(|\mathscr{P}|-1)$ submatrix can represent all nonzero vectors in the vector space
$\mathbb{F}_{2}^{|\mathcal{P}|}$. An example of $\mathcal{M}(3)$ for $\widetilde{\mathbf{M}}$ in (8) is as follows:

$$
\mathcal{M}(3)=\left[\begin{array}{ccccccc}
1 & 1 & 0 & 0 & 1 & 0 & 1 \\
1 & 1 & 0 & 1 & 0 & 1 & 0 \\
1 & 0 & 1 & 0 & 0 & 1 & 1 \\
-1 & -1 & 0 & -1 & 1 & -1 & \frac{1}{1} \\
1 & 1 & 1 & 0 & 1 & 1 & 1 \\
1 & 1 & 1 & 1 & 0 & 1 & 1 \\
1 & 1 & 1 & 1 & 1 & 1 & 1
\end{array}\right]
$$

Lemma 1: Let $\mathcal{M}(|\mathcal{P}|)$ be an auxiliary matrix of a type 2 modified path-link matrix $\widetilde{\mathbf{M}}$ with an end-to-end path set $\mathcal{P}$. Then, $\operatorname{rank}(\mathcal{M}(|\mathcal{P}|))=2^{|\mathcal{P}|}-1$, i.e., all $2^{|\mathcal{P}|}-1$ column vectors in $\mathcal{M}(|\mathcal{P}|)$ are linearly independent.

The proof of Lemma 1 is showed in Appendix A. With Lemma 1, the following theorem gives the rank of a type 2 modified path-link matrix.

Theorem 2: Let a directed acyclic graph $\mathcal{G}=(\mathcal{V}, \mathcal{E})$ be given with a system of linear equations in matrix form $\widetilde{\mathbf{M}} \hat{\mathbf{a}}=$ $\hat{\mathbf{c}}$. Then, $\operatorname{rank}(\widetilde{\mathbf{M}})=\left|\mathcal{E}_{I} \cup \mathcal{E}_{V}\right|$.

Proof: Let $\mathcal{M}(|\mathcal{P}|)$ be an auxiliary matrix of $\widetilde{\mathbf{M}}$. The $\left|\mathcal{E}_{I} \cup \mathcal{E}_{V}\right|$ column vectors in $\widetilde{\mathbf{M}}$ are among the $2^{|\mathcal{P}|}-1$ column vectors in $\mathcal{M}(|\mathcal{P}|)$. By Lemma 1 , all $2^{|\mathcal{P}|}-1$ column vectors in $\mathcal{M}(|\mathcal{P}|)$ are linearly independent. Thus, these $\left|\mathcal{E}_{I} \cup \mathcal{E}_{V}\right|$ column vectors in $\widetilde{\mathbf{M}}$ are also linearly independent. As a result, $\operatorname{rank}(\widetilde{\mathbf{M}})=\left|\mathcal{E}_{I} \cup \mathcal{E}_{V}\right|$.

Corollary 1: Let $\widetilde{\mathbf{M}} \hat{\mathbf{a}}=\hat{\mathbf{c}}$ be given. Then, $\hat{\mathbf{a}}$ can be determined by least-squares.

Proof: When the number of equations is equal to the number of unknowns, i.e., $|\mathscr{P}|-1=\left|\mathcal{E}_{I} \cup \mathcal{E}_{V}\right|, \widetilde{\mathbf{M}}$ is a square matrix. Theorem 2 ensures that $\widetilde{\mathbf{M}}$ is invertible. Thus, â can be determined as

$$
\hat{\mathbf{a}}=\widetilde{\mathbf{M}}^{-1} \hat{\mathbf{c}} .
$$

When the number of equations is greater than the number of unknowns, i.e., $|\mathscr{P}|-1>\left|\mathcal{E}_{I} \cup \mathcal{E}_{V}\right|$, the system is over-determined. We can apply least-squares [13] to obtain an approximate solution which minimizes the residual error $\|\hat{\mathbf{c}}-\mathbf{M} \hat{\mathbf{a}}\|$. Theorem 2 ensures that $\widetilde{\mathbf{M}}^{\mathrm{T}} \widetilde{\mathbf{M}}$ is invertible. Thus, â can be determined as

$$
\hat{\mathbf{a}}=\left(\widetilde{\mathbf{M}}^{\mathrm{T}} \widetilde{\mathbf{M}}\right)^{-1} \widetilde{\mathbf{M}}^{\mathrm{T}} \hat{\mathbf{c}} .
$$

We note that (14) is a special case of (15).

The following theorem proves the consistency of the corresponding estimators.

Theorem 3: $1-\hat{\alpha}_{j}$ is a consistent estimator of $1-\alpha_{j}$.

Proof: For each $e_{j} \in \mathcal{E}_{I} \cup \mathcal{E}_{V}, 1-\hat{\alpha}_{j}$ is a function of $\hat{\alpha}_{j}$, while $\hat{\alpha}_{j}$ is a function of $\hat{\theta}_{1}, \hat{\theta}_{2}, \ldots, \hat{\theta}_{|\mathscr{P}|-1}$. Since $\hat{\theta}_{i} \stackrel{p}{\rightarrow} \theta_{i}$, the continuous mapping theorem and Slutsky's theorem [14] yield that $1-\hat{\alpha}_{j} \stackrel{p}{\rightarrow} 1-\alpha_{j}$, where $\stackrel{p}{\rightarrow}$ denotes convergence in probability.

Proposition 2: The loss rate of a link can be consistently estimated if and only if the link is an identifiable link.

Proof: Theorem 3 shows that the loss rate of all identifiable links can be consistently estimated by the estimators, which together with Proposition 1, proves Proposition 2. 


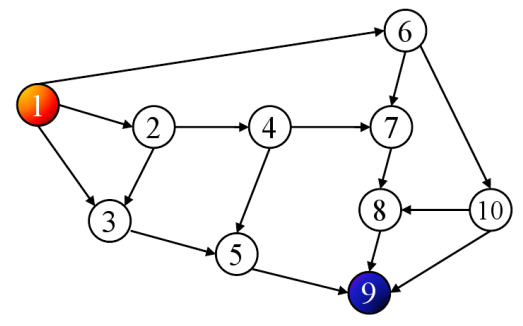

(a) One source.

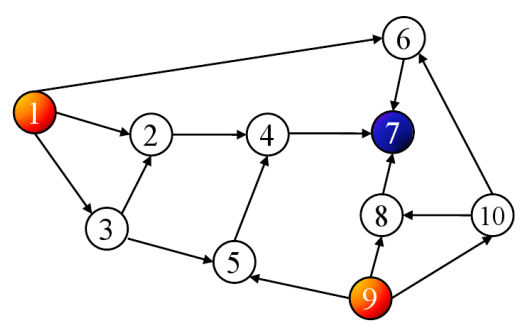

(b) Two sources

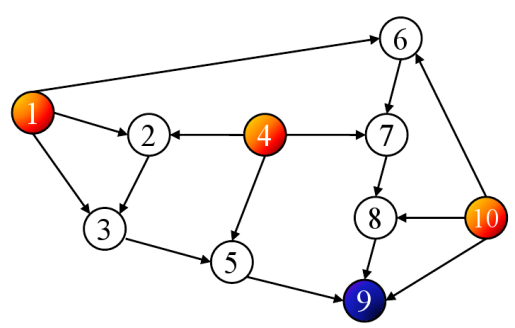

(c) Three sources.

Fig. 2. Directed acyclic graphs with different number of sources. (a) One source (node 1) and one receiver (node 9); (b) two sources (nodes 1 and 9) and one receiver (node 7); (c) three sources (nodes 1,4 and 10) and one receiver (node 9).

\section{Performance Evaluation}

\section{A. Simulation Setup}

In each simulation, we obtain an estimate $1-\hat{\alpha}_{j}$ of the actual link loss rate $1-\alpha_{j}$ for the $j$ th link in $\mathcal{E}_{I} \cup \mathcal{E}_{V}$. The root mean square error (RMSE) is used to determine the estimation accuracy across all identifiable links. RMSE is computed as

$$
\operatorname{RMSE}=\left(\sum_{j=1}^{\left|\mathcal{E}_{I}\right|} \frac{\left|\alpha_{j}-\hat{\alpha}_{j}\right|^{2}}{\left|\mathcal{E}_{I}\right|}\right)^{1 / 2} .
$$

For the network topology, we consider the Internet2 Network Map [15], which is a high-performance backbone network created by the Internet 2 community. The topology is modified as the one used in [10], consisting of 10 nodes and 15 edges. We apply the orientation algorithm [10] that converts the modified topology with selected sources to three directed acyclic graphs with different number of sources in Fig. 2, where all links are identifiable.

A random link loss rate $1-\alpha_{j}$ is assigned to the $j$ th link in $\mathcal{E}_{I} \cup \mathcal{E}_{V}$, where $\alpha_{j}$ is uniformly distributed in $\left[\alpha_{\text {ave }}-\right.$ $\left.0.05, \alpha_{a v e}+0.05\right]$. The value of $\alpha_{\text {ave }}$ is chosen as $0.7,0.75$, $0.8,0.85,0.9$, and 0.95 , to adjust the average success rate across all links. After assigning each link a loss rate, we send

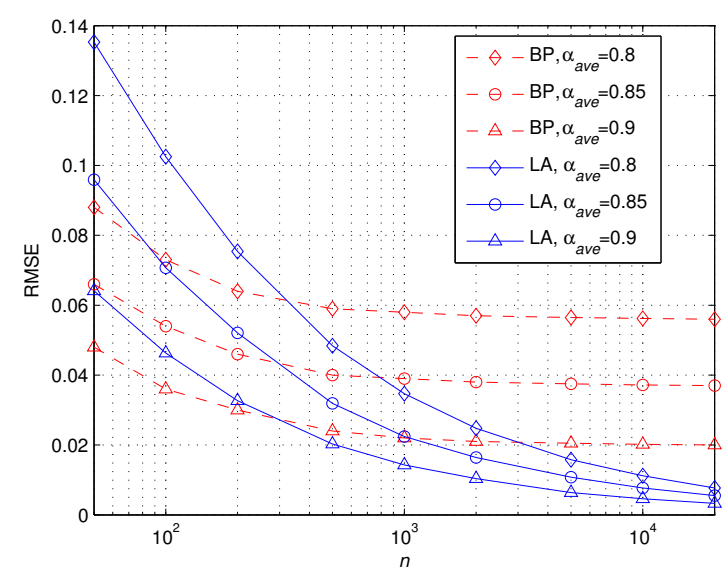

Fig. 3. RMSE of BP and LA, versus the number of probe batches $n$, for different $\alpha_{\text {ave }}$.

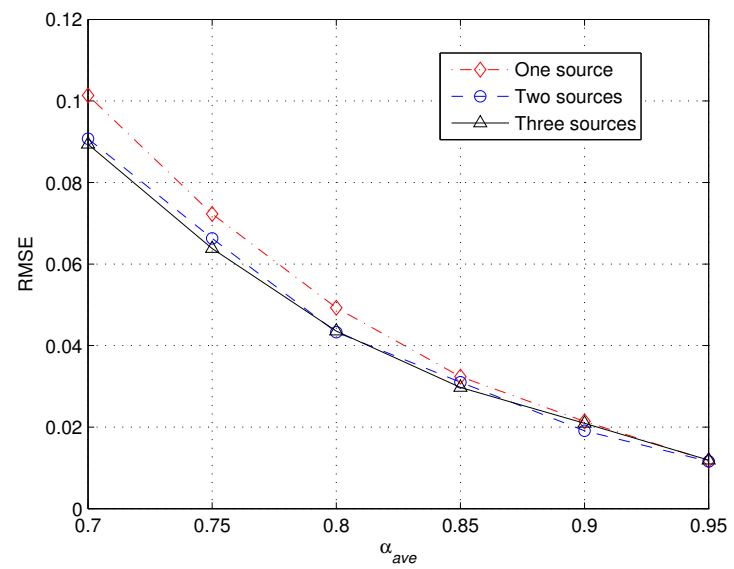

Fig. 4. RMSE of LA, versus the average success rate $\alpha_{a v e}$, for different number of sources.

$n$ batches of probe packets. Each probe traversing a link is dropped at a fixed probability as the link loss rate.

We use our proposed linear algebraic (LA) approach and the belief propagation (BP) algorithm [10] to infer loss rates, respectively. The results are averaged over 100 simulations to eliminate possible random effects, where each simulation has new loss rate assignments and new loss processes.

\section{B. Simulation Results}

First, we compare the estimation accuracy using LA and BP. Fig. 3 shows the RMSE as a function of the number of probe batches, for different average link success rates, based on the graph with one source in Fig. 2(a). It shows that LA achieves better accuracy than BP, after sending reasonably sufficient probe batches $(n>400)$.

Fig. 4 shows the RMSE as a function of the average success rate, for the graphs with different number of sources in Fig. 2, with 500 probe batches. Results show that more probe batches are required to achieve the same estimation accuracy in the networks with smaller average success rates.

Moreover, comparing the relative position of the three curves in Fig. 4 and their corresponding topologies, we obtain the following observations: Fig. 2(c) with three sources 
achieves better estimation accuracy than Fig. 2(a) with one source; Fig. 2(b) with two sources achieves similar accuracy as Fig. 2(c) with three sources but they have different locations of receiver. It shows that both the number of sources and the locations of sources/receiver affect the estimation accuracy, but the effect is negligible with relatively large success rates or sufficient probe batches. Thus, we can use a small number of sources and flexibly choose their locations in practice.

\section{CONCLUSION}

In this paper, we investigated active loss tomography on mesh topologies. We established a lower bound on probe size for obtaining valid end-to-end observations when network coding is applied. We proposed a linear algebraic (LA) approach and developed consistent estimators of link loss rates. Simulation results showed that the LA approach achieves better estimation accuracy than the BP algorithm, after sending reasonably sufficient probes. Future work includes formulating the procedure of finding a valid probe coding scheme with minimal probe size.

\section{APPENDIX}

\section{A. Proof of Lemma 1}

We prove it by induction. We mention that the matrix $\mathcal{M}(k)$ have binary entries and the column vectors are defined in a vector space over a finite field $\mathbb{F}_{2}$. It can be verified that $\mathcal{M}(2)$ has full rank. Assume that matrix $\mathcal{M}(k)$ also has full rank. That is, all $2^{k}-1$ columns in $\mathcal{M}(k)$ are linearly independent. Thus, the modulo 2 summation of any $m$ columns of this matrix, for $m=2, \ldots, 2^{k}-1$, has at least one nonzero entry. Now, consider matrix $\mathcal{M}(k+1)$. This matrix can be represented as follows after row and column permutations:

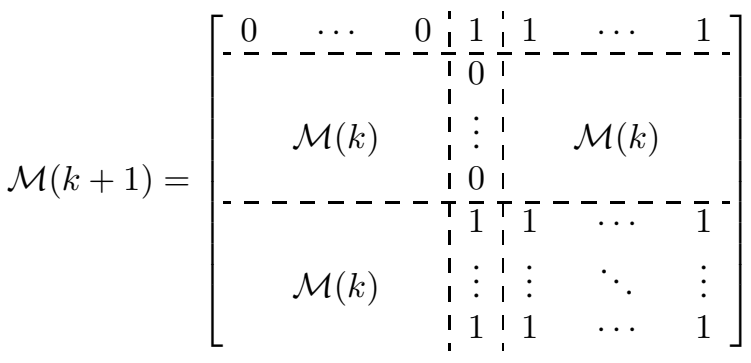

Permutations would not change its rank. The top row represents the new added path, followed by two submatrices $\mathcal{M}_{1}=[\mathcal{M}(k) \mathbf{0} \mathcal{M}(k)]$ and $\mathcal{M}_{2}=[\mathcal{M}(k) \mathbf{1}]$, where $\mathbf{0}$ and 1 are columns of 0 and 1 , respectively. We note that the path sets of $\mathcal{M}_{1}$ (rows in $\mathcal{M}_{1}$ ) do not include the new added path, while those of $\mathcal{M}_{2}$ all include it. Now, we show that the matrix $\mathcal{M}(k+1)$ has full rank. To do so, we show that the summation of all possible combinations of these $2^{k+1}-1$ columns in $\mathcal{M}(k+1)$ is a nonzero vector (i.e., there exists at least one nonzero entry in the summation vector).

First, the middle column $\left[\begin{array}{lll}1 & 0 & 1\end{array}\right]^{\mathrm{T}}$ is included in the combination of the columns that we choose. Since the entries of the last row in $\mathcal{M}(k)$ are all ones, in the summation of the chosen vectors, at least one entry would be nonzero. This entry corresponds to the last row in $\mathcal{M}_{1}$ or in $\mathcal{M}_{2}$. From now on, we exclude the middle column from our choices.
Second, we choose the columns from either the $2^{k}-1$ columns on the left-hand side or the $2^{k}-1$ columns on the right-hand side (not both at the same time). In this case, at least one entry in the summation vector would be nonzero corresponding to the rows in $\mathcal{M}_{1}$. It is because of the linear independency of the columns in $\mathcal{M}(k)$.

Third, we choose the columns from both the $2^{k}-1$ columns on the left-hand side and on the right-hand side. In this case, if an odd number of columns is chosen from the the right-hand side, the entry of the summation vector corresponding to the top row would be nonzero. However, if an even number of columns is chosen from the right-hand side, at least one entry of the summation vector corresponding to the rows in $\mathcal{M}_{2}$ would be nonzero, because of the linear independency of the columns in $\mathcal{M}(k)$. To this end, we have considered the modulo 2 summation for all possible combinations of the columns in matrix $\mathcal{M}(k+1)$, and there is always at least one nonzero entry in the summation vector. Therefore, all these $2^{k+1}-1$ column vectors in $\mathcal{M}(k+1)$ are linearly independent.

\section{ACKNOWLEDGEMENT}

This research is supported by the Natural Sciences and Engineering Research Council (NSERC) of Canada under grant number STPSC 356767.

\section{REFERENCES}

[1] R. Castro, M. Coates, G. Liang, R. Nowak, and B. Yu, "Network tomography: Recent developments," Statistical Science, vol. 19, no. 3, pp. 499-517, Aug. 2004.

[2] A. Chen, J. Cao, and T. Bu, "Network tomography: Identifiability and fourier domain estimation," in Proc. of IEEE INFOCOM, Anchorage, AK, May 2007.

[3] P. Sattari, A. Markopoulou, and C. Fragouli, "Multiple source multiple destination topology inference using network coding," in Proc. of NetCod Workshop, Lausanne, Switzerland, June 2009.

[4] R. Caceres, N. Duffield, J. Horowitz, and D. Towsley, "Multicast-based inference of network-internal loss characteristics," IEEE Trans. Inform. Theory, vol. 45, no. 7, pp. 2462-2480, Nov. 1999.

[5] M. Coates and R. Nowak, "Network loss inference using unicast end-toend measurement," in Proc. of ITC Seminar on IP Traffic, Measurement and Modelling, Monterey, CA, Sept. 2000.

[6] W. Zhu and Z. Geng, "A bottom-up inference of loss rate," Computer Communications, vol. 28, no. 4, pp. 351-365, Mar. 2005.

[7] T. Bu, N. Duffield, F. Presti, and D. Towsley, "Network tomography on general topologies," in Proc. of ACM SIGMETRICS, Marina Del Rey, CA, June 2002.

[8] R. Ahlswede, N. Cai, S. Li, and R. Yeung, "Network information flow," IEEE Trans. Inform. Theory, vol. 46, no. 4, pp. 1204-1216, July 2000.

[9] C. Fragouli, A. Markopoulou, R. Srinivasan, and S. Diggavi, "Network monitoring: It depends on your points of view," in Proc. of ITA Workshop, San Diego, CA, Jan. 2007.

[10] M. Gjoka, C. Fragouli, P. Sattari, and A. Markopoulou, "Loss tomography in general topologies with network coding," in Proc. of IEEE GLOBECOM, Washington, DC, Nov. 2007.

[11] Y. Mao, F. Kschischang, B. Li, and S. Pasupathy, "A factor graph approach to link loss monitoring in wireless sensor networks," IEEE J. Select. Areas Commun., vol. 23, no. 4, pp. 820-829, Apr. 2005.

[12] B. Sun and Z. Zhang, "Probabilistic diagnosis of link loss using end-toend path measurements and maximum likelihood estimation," in Proc. of IEEE ICC, Dresden, Germany, June 2009.

[13] R. Myers, D. Montgomery, and G. Vining, Generalized Linear Models: With Applications in Engineering and the Sciences. John Wiley \& Sons, Inc., 2001.

[14] G. Grimmett and D. Stirzaker, Probability and Random Processes, 3rd ed. Oxford University Press, 2001.

[15] "Internet2 network," http://www.internet2.edu/network/. 